\title{
Associação entre prevalência de dor musculoesquelética e variáveis do treinamento de força
}

\section{Association between prevalence of musculoskeletic pain and strength training variables}

\section{Asociación entre prevalencia de dolor musculoesquelético y variable del entrenamiento de fuerza}

\author{
(D) Carlos Leandro Tiggemann \\ Universidade do Vale do Taquari - UNIVATES, Lajeado, RS - Brasil \\ cltiggemann@univates.br \\ (D) Camila Zanatta \\ Universidade do Vale do Taquari - UNIVATES, Lajeado, RS - Brasil \\ camii.zanatta@yahoo.com.br \\ (D) Cleber Cremonese \\ Universidade Federal da Bahia, Salvador (BA), Brasil. \\ clebercre@yahoo.com.br \\ D) Jéssica Luana Dornelles da Costa \\ Universidade do Vale do Taquari - UNIVATES, Lajeado, RS - Brasil \\ jluana@univates.br \\ (D) Caroline Pietta Dias \\ Universidade Federal do Rio Grande do Sul, Porto Alegre/RS, Brasil \\ carolpieta@yahoo.com.br
}

Resumo: O objetivo foi medir a prevalência de dor musculoesquelética em praticantes de treinamento de força (TF) e sua associação com diferentes variáveis do treino. A amostra foi composta por 175 adultos praticantes de TF. Foram utilizados como instrumentos de avaliação um questionário geral e o Questionário Nórdico de Dor. Os resultados apre- sentaram que $73,7 \%$ da amostra investigada indicaram dor em, pelo me- nos, um ponto anatômico. Em relação às variáveis do TF, os sujeitos que praticam treinamento há mais tempo ( $>3$ anos) e que utilizaram cargas moderadas ou pesadas apresentaram maior prevalência de dor quando 
comparado aos seus pares. Conclui-se, portanto, que a prevalência de dor entre praticantes de TF é elevada, uma vez que o tempo de prática e o uso de cargas leves potencializam sua ocorrência.

Palavras-chave: Treinamento de Resistência. Dor. Força Muscular.

\begin{abstract}
The objective was to measure the prevalence of musculoskeletal pain in strength training practitioners (ST) and their association with different training variables. The sample consisted of 175 practicing adults. A general questionnaire and the Nordic Pain Questionnaire were used as evaluation instruments. The results showed that $73.7 \%$ of the investigated sample indicated pain in at least one anatomic site. In relation to the variables of the ST, those subjects who had trained a longer period of time (> 3 years) and who used moderate or high loads, presented higher prevalence of pain when compared to their peers. It is concluded that the prevalence of pain among ST practitioners is high, where the time of practice and the use of light loads increases its occurrence.
\end{abstract}

Key words: Resistance Training. Pain. Muscle Strength.

Resumen: El objetivo fue medir prevalencia de dolor musculoesquelético en practicantes de entrenamiento de fuerza (EF) y asociación con diferentes variables del entrenamiento. Muestra fue compuesta por 175 adultos practicantes de EF. Se utilizaron como instrumentos de evaluación un cuestionario general y el Cuestionario Nórdico de Dolor. Los resultados mostraron que el $73,7 \%$ de la muestra indicó dolor en al menos un punto anatómico. En cuanto a las variables del EF, aquellos sujetos que entrenaron hace más tiempo ( $>3$ años) y que utilizaron cargas moderadas o pesadas, presentaron mayor prevalencia de dolor cuando comparado a sus pares. Se concluye que la prevalencia de dolor entre practicantes de TF es elevada, donde el tiempo de práctica y el uso de cargas leves potencializa su ocurrencia.

Palabras-clave: Entrenamiento de Resistencia. Dolor. Fuerza muscular.

Submetido em: 24/11/2018

Aceito em: 05/09/2019 


\section{Introdução}

Recentemente, o Instituto Brasileiro de Geografia e Estatística (IBGE) divulgou dados sobre o sedentarismo e prática de atividades físicas no Brasil (IBGE, 2017). Os dados indicam que 62,1\% das pessoas acima de 15 anos não praticam qualquer tipo de esporte ou atividades físicas, sendo elas, portanto, consideradas sedentárias. O estudo ainda apontou que entre os 28,1 milhões de praticantes de alguma atividade física, 49,1\% são praticantes da caminhada, $16,8 \%$ de fitness ou academia e $7,2 \%$ de musculação ou culturismo. O Colégio Americano de Medicina do Esporte (ACSM, 2018) recomenda a prática regular de atividades físicas, dando especial atenção aos exercícios físicos de força muscular, de flexibilidade e neuromotores direcionados ao sistema cardiorrespiratório. Tais recomendações são baseadas nos inúmeros benefícios que esses exercícios proporcionam à saúde humana, contribuindo de forma específica a sujeitos portadores de doenças psicológicas, neurológicas, metabólicas, cardiovasculares, pulmonares, câncer e musculoesqueléticas (ACSM, 2018; PEDERSEN e SALTIN, 2015).

Em relação aos distúrbios musculoesqueléticos, sabe-se que o músculo desempenha importante papel protetor das estruturas passivas das diferentes articulações, sendo que a permanência em determinadas posições por tempos prolongados, a hipotonicidade proveniente do desuso, ou mesmo a fadiga pelo gesto repetitivo, podem causar uma transferência excessiva de carga àquelas estruturas, provocando dor (COSTA e PALMA, 2005). A dor possui causas multifatoriais, acometendo várias regiões do corpo humano, comprometendo direta e decisivamente a funcionalidade das atividades do dia a dia, sendo que diferentes estudos têm buscado identificar a associação da dor muscular como causadora ou como consequência de diferentes desvios posturais e lesões músculo-articulares (BUTRAGUEÑO, BENITO e MAFFULLI, 2014; MELLO et al., 2014). 
No que se refere ao treinamento de força (TF), as lesões músculo-articulares referem-se a desordens agudas e crônicas que se manifestam com a dor ou podem interferir no desempenho do treinamento (KOLBER et al., 2010). Em estudo epidemiológico realizado entre os anos de 1990 e 2007, nos Departamentos de Emergência dos Estados Unidos (KERR et al., 2010), foram identificadas mais de 25 mil lesões decorrentes do TF, ocorrendo na grande maioria entre os homens (82\%), em especial na parte superior do tronco, incluindo ombros $(25,3 \%)$ e região inferior do tronco $(19,7 \%)$. O mecanismo mais comum de lesão foi a queda dos pesos sobre os sujeitos (65,5\%), sendo que lesões mais frequentes ocorreram por meio do uso de pesos livres $(90,4 \%)$. Em estudo realizado com praticantes de musculação da cidade de Curitiba, Brasil, foi identificada uma ocorrência de $44,4 \%$ de sujeitos acometidos por lesões decorrentes do TF (SOUZA, MOREIRA e CAMPOS, 2015). Ombros (35\%) e joelhos (30\%) foram os locais mais afetados, sendo a distensão muscular o tipo de lesão mais encontrada (35\%), seguido pela tendinopatia (25\%) e a dor inespecífica (20\%).

Embora a associação entre a prática do TF ou dos níveis de força muscular com diferentes parâmetros da postura ou de desvios posturais seja controversa na literatura científica (TIGGEMANN et al., 2018), ela não pode ser desconsiderada como fator de promoção das dores músculos esqueléticas. Baroni et al. (2010) avaliaram as alterações posturais em 306 praticantes de TF de ambos os sexos, na faixa etária entre 14 e 73 anos da cidade de Caxias do Sul, Brasil. Os resultados indicaram uma grande prevalência de sujeitos com algum tipo de alteração na coluna cervical (43,4\%), torácica $(55,2 \%)$ e lombar $(73,8 \%)$, sendo que $81 \%$ apresentaram ombros anteriorizados. Embora a prevalência de desvios posturais pareça ser considerável, poucos estudos associaram esse aspecto à ocorrência de dores músculo-articulares. Mello et al. (2014) investigaram a associação da presença de discinese escapular (i.e., alterações na posição e nos movimentos da escápula em relação à caixa torácica) com a sintomalogia dolorosa de ombro em praticantes de TF. Dos 35 sujeitos avaliados, 30 apresentaram discinese 
Associação entre prevalência de dor musculoesquelética e variáveis...

Carlos Leandro Tiggemann . Camila Zanatta • Cleber Cremonese • Jéssica Luana Dornelles da Costa

escapular, sendo que uma relação significativa foi encontrada com a presença de dor durante os esforços.

A presença de dores por praticantes de TF é um aspecto comprometedor no desenvolvimento de seus treinamentos repercutindo em seu desempenho e sua saúde, causando limitações e desconfortos consideráveis, podendo levar os sujeitos ao afastamento da prática da modalidade (COSTA; PALMA, 2005). Poucos estudos foram encontrados relatando a prevalência de dores musculoesqueléticas em praticantes de TF. Oliveira e Casa Júnior (2014) identificaram 79\% de sua amostra de praticantes de TF com dor lombar, sendo que destes apenas $11 \%$ foram classificadas com incapacidade moderada. Oliva, Bankoff e Zamai (1998) identificaram que mais da metade da amostra ( $n=55$; homens e mulheres; 24 a 43 anos; praticantes de TF a pelo menos seis meses) do estudo (55\%) relatou dor decorrente exclusivamente da prática do TF, sendo a região do ombro a mais acometida (24\%).

Além disso, pouco se sabe sobre como as diferentes variáveis do TF (carga, velocidade de execução, séries, repetições, amplitude de movimento, entre outras) podem estar relacionadas à prevalência de dor em seus praticantes. Dessa forma, o objetivo do presente estudo foi medir a prevalência de dor musculoesquelética em praticantes de TF e sua associação com diferentes variáveis do treinamento.

\section{Procedimentos metodológicos}

A amostra foi composta por 175 adultos praticantes de TF, sendo selecionados de forma não probabilística por acessibilidade, advindos de três academias de musculação da cidade de Lajeado, Rio Grande do Sul, Brasil. Como critérios de inclusão, os indivíduos deveriam: estar realizando TF por um período superior a seis meses, responder negativamente a todas as questões do Questionário de Prontidão para Atividade Física (PAR-Q), não estar usando nenhum medicamento (à exceção de anticoncepcionais 
para as mulheres), não possuir histórico de cirurgia ou algum tipo de intervenção médica no sistema músculo-articular. Em relação aos aspectos éticos, a pesquisa foi aprovada pelo comitê de ética da Universidade do Vale do Taquari-RS (parecer no. 800.495), sendo que todos os participantes assinaram o termo de consentimento livre e esclarecido.

Como instrumentos de pesquisa foram utilizados um Questionário Geral e o Questionário Nórdico de Dor. Foram entregues 260 deles, sendo que destes 182 retornaram e sete foram excluídos por preenchimento incorreto, restando 175 questionários válidos para inclusão no estudo. Alguns questionários apresentaram questões não preenchidas, não invalidando as demais respostas para análise. O Questionário Geral foi composto por questões contemplando as características pessoais e as do TF realizado nos últimos seis meses, sendo todas as questões elaboradas por meio de respostas de múltipla escolha. Esse questionário passou por um processo de validação prévia quanto a seu conteúdo, sendo avaliado por quatro pesquisadores experientes e de comprovado reconhecimento na área, por meio de duas rodadas de avaliação. Também a capacidade de reprodutibilidade foi avaliada, sendo aquele questionário aplicado e replicado em um intervalo de uma semana, para 25 sujeitos não pertencentes à amostra do presente estudo, respeitando os mesmos critérios de inclusão. Valores de correlação intraclasse significativos $(p<0,05)$ foram encontrados em todas as questões, sendo que $13 \%$ das questões apresentaram um valor de $r$ variando de 0,700 a $0,800,50 \%$ das questões entre 0,801 a 0,900, e 37\% das questões com valor superior a 0,901.

Quinze questões avaliavam informações específicas do TF, são elas: 1) tempo de prática, sendo desconsideradas as interrupções inferiores há quatro semanas (por motivos de férias, doenças, viagens, outros); 2) objetivo do treinamento, sendo as respostas agrupadas em saúde/força, hipertrofia e outros (emagrecimento, fins esportivos, terapêutico, outros); 3) frequência semanal habitual do TF (número de sessões na semana); 4) realização de algum tipo de exercício de aquecimento específico (esteira, bicicleta, exercícios 
localizados); 5) realização de exercícios de alongamento; 6) intervalo entre as séries e os exercícios - em minutos; 7) soma das séries realizadas de todos os exercícios envolvendo membros superiores e tórax (bíceps, tríceps, peito, costas, ombros/trapézio) durante a semana; 8) soma das séries realizadas de todos os exercícios envolvendo membros inferiores e abdômen (abdominal, extensores de coluna, coxas anterior e posterior, panturrilha) durante a semana; 9) número médio de repetições realizadas por série na maioria dos exercícios; 10) carga utilizada durante as séries na maioria dos exercícios; 11) velocidade de execução aproximada para cada repetição nos diferentes exercícios; 12) amplitude articular realizada na maioria dos exercícios (completa = máxima amplitude articular; parcial = amplitude inferior à completa); 13) amplitude articular específica aos exercícios agachamento/leg press (e outros similares), sendo considerada como parcial aquela com um ângulo aproximado de $90^{\circ}$ do joelho, e a completa aquela além deste valor (flexão mais acentuada); 14) forma de realização do exercício puxada polia alta (anterior $=$ pela frente da cabeça; posterior $=$ por trás da cabeça); 15) forma de realização do exercício desenvolvimento (anterior = pela frente da cabeça; posterior = por trás da cabeça).

O Questionário Nórdico de Dor foi validado e traduzido para a língua portuguesa e refere-se à avaliação de distúrbios musculoesqueléticos (BARROS; ALEXANDRE, 2003). Esse questionário apresenta o desenho do corpo humano com as regiões grifadas e especificadas (pescoço, ombros, parte superior das costas, cotovelos, punhos/mãos, parte inferior das costas, quadril/coxas, joeIhos, tornozelos/pés), possuindo as seguintes questões a serem respondidas: (1) ocorrência de dor, formigamento e dormência, o indivíduo assinala isso considerando os últimos doze meses; (2) se o indivíduo deixou de realizar alguma de suas atividades diárias, trabalho, atividades domésticas e de lazer, por dor em alguma das regiões acima citadas; (3) se o indivíduo buscou atendimento de algum profissional da área da saúde em 12 meses devido à referida condição; (4) se houve alguma ocorrência nos últimos sete dias 
Associação entre prevalência de dor musculoesquelética e variáveis...

Carlos Leandro Tiggemann . Camila Zanatta • Cleber Cremonese • Jéssica Luana Dornelles da Costa

anteriores à entrevista. Todas as questões permitem apenas as respostas sim ou não.

Para a análise, foram utilizados apenas os dados da dor referida nos últimos 12 meses por representar um comportamento crônico de dor, podendo ser entendido como mais representativo do aspecto do treinamento. Além disso, somente os dados das regiões anatômicas dos ombros, da região inferior das costas e dos joelhos foram utilizados, por apresentarem as maiores prevalências (acima de 30\%) e por ser representativo das três regiões anatômicas (membro superior, inferior e tronco). Não foram encontradas diferenças nas proporções de relato de dor entre homens e mulheres e, portanto, foram analisadas conjuntamente. A construção do banco de dados e as análises estatísticas foram realizadas por meio do programa SPSS Statistic Data 20 (Statistical Package for Social Sciences - Chicago, IL, 2008). A análise descritiva das variáveis avaliadas foi realizada por meio de valores absolutos, frequências e prevalências do desfecho. A análise bruta, aplicando Regressão de Poisson, apresentou as Razões de Prevalência (RP) e respectivos intervalos de confiança de 95\% (IC95\%). Para identificar associação entre os desfechos (dor referida) e as variáveis de exposição (variáveis do TF), aplicou-se o teste qui-quadrado para heterogeneidade, considerado um nível de significância de $5 \%(p \leq 0,05)$.

\section{Resultados}

A amostra foi constituída por sujeitos do sexo feminino (44\%) e masculino (56\%), com idade média de 36,2 $\pm 13,1$ anos, massa corporal de $71,0 \pm 12,0 \mathrm{~kg}$ e índice de massa corporal 24,1 $\pm 2,7 \mathrm{~kg}$ / $\mathrm{m}^{2}$. Referente à prevalência de dor musculoesquelética, 73,7\% da amostra investigada indicaram dor em, pelo menos, um ponto anatômico (dado não apresentado em tabela). As regiões relatadas com maior prevalência de dor nos últimos 12 meses foram na região inferior das costas $(38,3 \%)$, dos ombros $(36,6 \%)$ e dos joe- 
Associação entre prevalência de dor musculoesquelética e variáveis...

Carlos Leandro Tiggemann . Camila Zanatta • Cleber Cremonese • Jéssica Luana Dornelles da Costa

Ihos (31,4\%). Entre 8,0 a 9,7\% dos sujeitos relataram que a dor os impediu de realizar atividades diárias, sendo que o percentual dos que procuraram ajuda de algum profissional da saúde no último ano por causa da sua condição variou de 8,6 a 13,7\%. Apenas 6,3 a $13 \%$ dos sujeitos relataram dor nos últimos sete dias (tabela 1 ).

Tabela 1 - Prevalência de dores conforme região anatômica [n (\%)]. Total de 175 sujeitos.

\begin{tabular}{lcccc}
\hline Região anatômica & $\begin{array}{c}\text { Dor últimos 12 } \\
\text { meses }\end{array}$ & $\begin{array}{c}\text { Dor impediu de } \\
\text { realizar atividades }\end{array}$ & $\begin{array}{c}\text { Dor levou a consultara } \\
\text { profissional de saúde }\end{array}$ & $\begin{array}{c}\text { Dor últimos 7 } \\
\text { dias }\end{array}$ \\
\hline Pescoço & $49(28,0)$ & $4(2,3)$ & $10(5,7)$ & $8(4,6)$ \\
Ombros & $64(36,6)$ & $14(8,0)$ & $15(8,6)$ & $15(8,6)$ \\
Parte superior costas & $49(28,0)$ & $6(3,4)$ & $11(6,3)$ & $8(4,6)$ \\
Cotovelos & $18(10,3)$ & $2(1,1)$ & $5(2,9)$ & $7(4,0)$ \\
Punhos/Mãos & $38(21,7)$ & $9(5,1)$ & $5(2,9)$ & $8(4,6)$ \\
Parte inferior costas & $67(38,3)$ & $15(8,6)$ & $24(13,7)$ & $23(13,0)$ \\
Quadril/Coxas & $30(17,1)$ & $7(4,0)$ & $11(6,3)$ & $8(4,6)$ \\
Joelhos & $55(31,4)$ & $17(9,7)$ & $21(12,0)$ & $11(6,3)$ \\
Tornozelos/Pés & $38(21,7)$ & $11(6,3)$ & $9(5,1)$ & $11(6,3)$ \\
\hline
\end{tabular}

Fonte: próprios autores.

A tabela 2 traz os dados referentes à associação da prevalência de dor musculoesquelética e variáveis do TF. Os resultados apresentaram associações estatisticamente significativas $(p \leq 0,05)$ apenas em duas variáveis: tempo de prática, em que os sujeitos que praticam TF há mais tempo (superior a três anos) apresentaram uma prevalência de dor no ombro 2,21 vezes maior (RP:2,21; IC95\% 1,34-3,67) e 62\% maior para dor na parte inferior das costas (RP:1,62; IC95\% 1,01-2,65), quando comparados aos de menor tempo desta prática ( $<1$ ano). Ainda, a carga utilizada apresentou a seguinte associação: aqueles indivíduos que treinam com cargas mais leves ou nenhuma possuem maior prevalência de dor nos ombros, quando comparados aos de maior carga (RP: 0,65; IC95\% $0,43-0,96)$. Nas demais variáveis não foram encontradas associações significativas com a dor musculoesquelética $(p>0,05)$. 
Associação entre prevalência de dor musculoesquelética e variáveis do treinamento de força

Carlos Leandro Tiggemann • Camila Zanatta • Cleber Cremonese · Jéssica Luana Dornelles da Costa

Tabela $2 a$ - Prevalência de dor e associação com as regiões anatômica, conforme variáveis do treinamento de força.

\begin{tabular}{|c|c|c|c|c|c|c|c|c|c|c|}
\hline Variáveis (n) & n (\%) & Ombro & $\begin{array}{l}\text { RP bruta } \\
\text { (IC95\%) }\end{array}$ & p-valor* & $\begin{array}{l}\text { Inferior } \\
\text { costas }\end{array}$ & $\begin{array}{l}\text { RP bruta } \\
\text { (IC95\%) }\end{array}$ & p-valor* & Joelhos & $\begin{array}{l}\text { RP bruta } \\
\text { (IC95\%) }\end{array}$ & p-valor* \\
\hline $\begin{array}{l}\text { 1) Tempo de prática } \\
\text { (174) }\end{array}$ & & & & 0,001 & & & 0,050 & & & 0,211 \\
\hline$<1$ ano & $57(32,8)$ & $14(24,6)$ & 1 & & $16(28,1)$ & 1 & & $21(36,8)$ & 1 & \\
\hline 1 a 3 anos & $49(28,2)$ & $13(26,5)$ & $1,08(0,56-2,07)$ & & $19(38,8)$ & $1,38(0,80-2,38)$ & & $16(32,7)$ & $0,88(0,52-1,50)$ & \\
\hline$>3$ anos & $68(39,1)$ & $37(54,4)$ & $2,21(1,34-3,67)$ & & $31(45,6)$ & $1,62(1,01-2,65)$ & & $18(26,5)$ & $0,72(0,43-1,21)$ & \\
\hline 2) Objetivo (174) & & & & 0,882 & & & 0,955 & & & 0,713 \\
\hline Saúde/força & $91(52,3)$ & $35(38,5)$ & 1 & & $35(38,5)$ & 1 & & $27(29,7)$ & 1 & \\
\hline Hipertrofia & $36(20,7)$ & $11(30,6)$ & $0,79(0,45-1,39)$ & & $13(36,1)$ & $0,94(0,57-1,56)$ & & $13(36,1)$ & $1,22(0,71-2,09)$ & \\
\hline Outros & $47(27,0)$ & $18(38,3)$ & $1,00(0,64-1,56)$ & & $18(38,3)$ & $1,00(0,64-1,56)$ & & $15(31,9)$ & $1,08(0,64-1,82)$ & \\
\hline $\begin{array}{l}\text { 3) Frequência sema- } \\
\text { nal (174) }\end{array}$ & & & & 0,284 & & & 0,676 & & & 0,973 \\
\hline $1-2 x$ & $70(40,2)$ & $29(41,4)$ & 1 & & $26(37,1)$ & 1 & & $23(32,9)$ & 1 & \\
\hline $3-4 x$ & $81(46,6)$ & $28(34,6)$ & $0,83(0,55-1,26)$ & & $30(37,0)$ & $1,00(0,66-1,51)$ & & $24(29,6)$ & $0,90(0,56-1,45)$ & \\
\hline$\geq 5 x$ & $23(13,2)$ & $7(30,4)$ & $0,73(0,37-1,45)$ & & $10(43,5)$ & $1,17(0,67-2,05)$ & & $8(34,8)$ & $1,06(0,55-2,04)$ & \\
\hline $\begin{array}{l}\text { 4) Aquecimento } \\
\text { (174) }\end{array}$ & & & & 0,290 & & & 0,225 & & & 0,706 \\
\hline Sim & $142(81,6)$ & $55(38,7)$ & 1 & & $51(35,9)$ & 1 & & $44(31,4)$ & 1 & \\
\hline Não & $32(18,4)$ & $9(28,1)$ & $0,73(0,40-1,31)$ & & $15(46,9)$ & $1,31(0,85-2,01)$ & & $11(34,4)$ & $1,11(0,65-1,90)$ & \\
\hline $\begin{array}{l}\text { 5) Alongamento } \\
\text { (174) }\end{array}$ & & & & 0,312 & & & 0,312 & & & 0,453 \\
\hline Antes & $19(10,0)$ & $10(52,6)$ & 1 & & $9(47,4)$ & 1 & & $8(42,1)$ & 1 & \\
\hline Após & $76(43,7)$ & $30(39,5)$ & $0,81(0,46-1,40)$ & & $29(38,2)$ & $0,81(0,46-1,40)$ & & $27(35,5)$ & $0,84(0,46-1,55)$ & \\
\hline Antes e após & $68(39,1)$ & $22(32,4)$ & $0,78(0,44-1,37)$ & & $25(36,8)$ & $0,78(0,44-1,37)$ & & $20(29,4)$ & $0,70(0,37-1,33)$ & \\
\hline Não & $11(6,3)$ & $2(18,2)$ & $0,58(0,20-1,70)$ & & $3(27,3)$ & $0,58(0,20-1,70)$ & & $0(0,0)$ & - & \\
\hline
\end{tabular}


Associação entre prevalência de dor musculoesquelética e variáveis do treinamento de força

Carlos Leandro Tiggemann • Camila Zanatta • Cleber Cremonese • Jéssica Luana Dornelles da Costa

Tabela 2b - Prevalência de dor e associação com as regiões anatômica, conforme variáveis do treinamento de força.

\begin{tabular}{|c|c|c|c|c|c|c|c|c|c|c|}
\hline Variáveis (n) & $\mathrm{n}(\%)$ & Ombro & RP bruta (IC95\%) & p-valor* & Inferior costas & RP bruta (IC95\%) & p-valor* & Joelhos & RP bruta (IC95\%) & p-valor* \\
\hline 6) Intervalo (175) & & & & 0,329 & & & 0,969 & & & 0,353 \\
\hline$\leq 1$ minuto & $112(64)$ & $38(33,9)$ & 1 & & $43(38,4)$ & 1 & & $38(33,9)$ & 1 & \\
\hline$>1$ minuto & $63(36)$ & $26(41,3)$ & $1,21(0,82-1,80)$ & & $24(38,1)$ & $0,99(0,67-1,47)$ & & $17(27,0)$ & $0,80(0,49-1,29)$ & \\
\hline $\begin{array}{l}\text { 7) Séries MS/ tórax } \\
\text { (174) }\end{array}$ & & & & 0,767 & & & 0,342 & & & - \\
\hline $10-20$ & $76(43,7)$ & $29(38,2)$ & 1 & & $27(35,5)$ & 1 & & - & - & \\
\hline $21-40$ & $65(37,4)$ & $22(33,8)$ & $0,89(0,57-1,39)$ & & $25(38,5)$ & $1,08(0,70-1,67)$ & & - & - & \\
\hline$\geq 40$ & $33(19,0)$ & $12(36,4)$ & $0,95(0,56-1,63)$ & & $15(45,5)$ & $1,28(0,79-2,07)$ & & - & - & \\
\hline $\begin{array}{l}\text { 8) Séries MI/ abdô- } \\
\text { men (175) }\end{array}$ & & & & - & & & 0,680 & & & 0,230 \\
\hline $10-20$ & $75(42,9)$ & - & - & & $31(41,3)$ & 1 & & $27(36,0)$ & 1 & \\
\hline $21-40$ & $64(36,6)$ & - & - & & $22(34,4)$ & $0,83(0,54-1,28)$ & & $19(29,7)$ & $0,82(0,51-1,34)$ & \\
\hline$\geq 40$ & $36(20,6)$ & - & - & & $14(38,9)$ & $0,94(0,58-1,54)$ & & $9(25,0)$ & $0,69(0,37-1,32)$ & \\
\hline $\begin{array}{l}\text { 9) Repetições } \\
\text { (174) }\end{array}$ & & & & 0,207 & & & 0,981 & & & 0,576 \\
\hline$\leq 6$ & $24(13,8)$ & $6(25,0)$ & 1 & & $9(37,5)$ & 1 & & $5(20,8)$ & 1 & \\
\hline 7-15 & $\begin{array}{c}131 \\
(75,3)\end{array}$ & $50(38,2)$ & $1,53(0,74-3,16)$ & & $51(38,9)$ & $1,03(0,59-1,81)$ & & $45(34,4)$ & $1,65(0,73-3,73)$ & \\
\hline$\geq 16$ & $19(10,9)$ & $8(42,1)$ & $1,68(0,70-4,03)$ & & $7(36,8)$ & $0,98(0,45-2,15)$ & & $5(26,3)$ & $1,26(0,43-3,74)$ & \\
\hline $\begin{array}{l}\text { 10) Carga } \\
\text { (175) }\end{array}$ & & & & 0,032 & & & 0,270 & & & 0,475 \\
\hline Nada/leve & $77(44,0)$ & $35(45,5)$ & 1 & & $33(42,9)$ & 1 & & $22(28,6)$ & 1 & \\
\hline Moderado/Alta & $98(56,0)$ & $29(29,6)$ & $0,65(0,43-0,96)$ & & $34(34,7)$ & $0,81(0,56-1,18)$ & & $33(33,7)$ & $1,18(0,75-1,85)$ & \\
\hline
\end{tabular}

$R P$ - Razão de Prevalência. IC - Intervalo de Confiança. *Teste qui-quadrado para heterogeneidade; Valores em negrito são estatisticamente significativos $(p \leq 0,05)$. 
Associação entre prevalência de dor musculoesquelética e variáveis do treinamento de força

Carlos Leandro Tiggemann • Camila Zanatta • Cleber Cremonese • Jéssica Luana Dornelles da Costa

Tabela 2c - Prevalência de dor e associação com as regiões anatômica, conforme variáveis do treinamento de força.

\begin{tabular}{|c|c|c|c|c|c|c|c|c|c|c|}
\hline Variáveis (n) & n (\%) & Ombro & RPbruta (IC95\%) & p-valor* & $\begin{array}{l}\text { Inferior } \\
\text { costas }\end{array}$ & RPbruta (IC95\%) & p-valor* & Joelhos & $\begin{array}{l}\text { RPbruta } \\
\text { (IC95\%) }\end{array}$ & p-valor* \\
\hline 11) Velocidade (174) & & & & 0,060 & & & 0,548 & & & 0,060 \\
\hline $1+1 s$ & $34(19,5)$ & $17(50,7)$ & 1 & & $16(47,1)$ & 1 & & $15(44,1)$ & 1 & \\
\hline $2+2 s$ & $113(64,9)$ & $40(35,4)$ & $0,71(0,47-1,08)$ & & $39(34,5)$ & $0,73(0,47-1,14)$ & & $34(30,1)$ & $0,68(0,43-1,09)$ & \\
\hline Outros & $27(15,5)$ & $7(25,9)$ & $0,52(0,25-1,07)$ & & $11(40,7)$ & $0,87(0,48-1,55)$ & & $6(22,2)$ & $0,50(0,23-1,12)$ & \\
\hline 12) Amplitude geral (175) & & & & 0,997 & & & 0,618 & & & 0,689 \\
\hline Parcial & $93(53,1)$ & $34(36,6)$ & 1 & & $34(36,6)$ & 1 & & $28(30,1)$ & 1 & \\
\hline Completa & $82(46,9)$ & $30(36,6)$ & $1,00(0,68-1,48)$ & & $33(40,2)$ & $1,10(0,76-1,60)$ & & $27(32,9)$ & $1,09(0,71-1,70)$ & \\
\hline $\begin{array}{l}\text { 13) Amplitude agac./leg } \\
\text { (173) }\end{array}$ & & & & - & & & 0,619 & & & 0,385 \\
\hline Parcial & $137(79,2)$ & - & - & & $51(37,2)$ & 1 & & $45(32,8)$ & 1 & \\
\hline Completa & $36(20,8)$ & - & - & & $15(41,7)$ & $1,12(0,72-1,75)$ & & $9(25,0)$ & $0,76(0,41-1,41)$ & \\
\hline $\begin{array}{l}\text { 14) Puxada polia alta } \\
\text { (148) }\end{array}$ & & & & 0,202 & & & & & & - \\
\hline Anterior & $78(52,7)$ & $23(29,5)$ & 1 & & $25(32,1)$ & 1 & 0,218 & - & - & \\
\hline Posterior & $13(8,8)$ & $3(23,1)$ & $0,78(0,27-2,24)$ & & $6(46,2)$ & $1,44(0,73-2,82)$ & & - & - & \\
\hline Ambas & $57(38,5)$ & $23(40,4)$ & $1,37(0,86-2,18)$ & & $24(41,1)$ & $1,31(0,84-2,05)$ & & - & - & \\
\hline $\begin{array}{l}\text { 15) Desenvolvi-mento } \\
\text { (112) }\end{array}$ & & & & 0,060 & & & 0,525 & & & - \\
\hline Anterior & $47(42)$ & $12(25,5)$ & 1 & & $16(34,0)$ & 1 & & - & - & \\
\hline Posterior & $13(7,4)$ & $4(30,8)$ & $1,21(0,46-3,13)$ & & $6(46,2)$ & $1,36(0,66-2,76)$ & & - & - & \\
\hline Ambos & $52(46,4)$ & $23(44,2)$ & $1,73(0,97-3,09)$ & & $21(40,4)$ & $1,19(0,71-2,00)$ & & - & - & \\
\hline
\end{tabular}

RP - Razão de Prevalência. IC - Intervalo de Confiança. *Teste qui-quadrado para heterogeneidade; Valores em negrito são estatisticamente significativos $(p \leq 0,05)$.

Fonte: próprios autores.

Revista Pensar a Prática. 2020, v.23:e55991 


\section{Discussão}

o objetivo do presente estudo foi medir a prevalência de dor musculoesquelética em praticantes de TF e sua associação com diferentes variáveis do treinamento. Três de cada quatro participantes investigados relataram pelo menos um ponto de dor nos últimos 12 meses $(73,7 \%)$, sendo que a dor na região inferior das costas $(38,3 \%)$, dos ombros $(36,6 \%)$ e dos joelhos $(31,4 \%)$ foram as de maior prevalência. De forma geral, essas dores musculoesqueléticas impediram apenas entre 8,0 a 9,7\% dos sujeitos de realizar atividades diárias, sendo que, aproximadamente, $7 \%$ procuraram ajuda de algum profissional da saúde para o tratamento delas. Alguns estudos também objetivaram investigar a prevalência de dores musculoesqueléticas em praticantes de TF. Rolla et al. (2004) também identificaram os mesmos locais que o do presente estudo como sendo os de maior prevalência. Dos 365 participantes investigados (55\% masculino; idade de 33,0 012,6 anos), $27 \%$ relataram a percepção de dor relacionada ao exercício físico realizado nas academias, sendo que joelhos (41\%), ombros (29\%) e coluna (20\%) foram as de maior prevalência. Oliveira e Casa Júnior (2014) realizaram um estudo com praticantes de TF associados unicamente a casos de lombalgias. Nesse estudo, dos 100 sujeitos praticantes de TF há mais de seis meses (homens e mulheres, idade de 28,7 78,7 anos), 79\% relataram dor lombar nos últimos 30 dias. Ainda, apenas $17 \%$ realizaram algum tipo de tratamento e $11 \%$ foram classificados com uma incapacidade moderada. Estima-se que cerca de $70 \%$ a $85 \%$ de toda a população mundial irão sentir dor lombar em alguma época de sua vida, sendo que as lombalgias são comuns na população, especialmente em países industrializados, como o Brasil (COSTA; PALMA, 2005).

Considerando apenas as regiões de maior prevalência de dor de nosso estudo, não foram encontradas diferenças significativas na proporção de sujeitos com dores musculoesqueléticas relativas aos diferentes objetivos pretendidos pelos participantes com o TF. Sabe-se que o objetivo do TF é um dos pilares para o direciona- 
mento da definição das variáveis que o compõem, ou seja, conforme as necessidades de cada indivíduo, treinamentos específicos são elaborados, podendo ser caracterizados por maiores ou menores volumes e intensidades (FLECK; KRAEMER, 2017). Fleck e Kraemer (2017) citam a frequência semanal, o número de séries e de repetições como as principais variáveis de volume, enquanto que as cargas utilizadas, a velocidade de execução, a ordem e seleção dos exercícios, a amplitude de execução e o intervalo entre as séries como sendo as variáveis de intensidade.

Sabe-se que o esforço repetitivo é um grande causador do aparecimento de desconfortos e dores musculoesqueléticas (GENEEN et al., 2017). Sendo assim, esse esforço repetitivo, de certa forma, é análogo e proporcional ao volume de um treinamento. Em relação às variáveis de volume analisadas separadamente, nosso estudo indicou que realizar uma quantidade de séries, repetições e dias de treinamento maiores não apresentou influência na prevalência de dor nos sujeitos. Entretanto, se considerarmos o tempo de prática como um somatório dessas variáveis, inclusive naquelas que representam a intensidade do treinamento, esta interpretação poderia ser diferenciada. Nesse sentido, o presente estudo constatou que os tempos de treinamento superiores há três anos repercutem em uma maior proporção de dores nos ombros e na parte inferior das costas, quando comparados com os menores tempos de prática. Assim, sabe-se que determinadas atividades com posições e movimentos frequentes realizados repetidamente, juntamente ao período e à sobrecarga de treinamento, podem provocar condições prejudiciais à saúde, tendo alto potencial de desequilíbrio muscular levando à dor (OLIVEIRA; CASA JÚNIOR, 2014).

A razão de prevalência de dor nas três regiões analisadas foi similar entre os sujeitos que realizavam ou não a prática de aquecimento antes dos treinamentos, bem como entre os que realizavam exercícios de alongamentos, independente do momento (antes ou após a sessão), ou de não realizá-los. A prática de alongamento e aquecimento, previamente aos exercícios, tem sido 
tradicionalmente indicada na preparação de sessões de treino, visando ao aumento da amplitude articular da região exercitada e à diminuição da rigidez muscular ou promoção do aumento da complacência muscular e, quando realizados conjuntamente, diminuem as chances de lesão dos grupos musculares envolvidos (LEWIS, 2014), sendo este entendimento não relacionado propriamente à sintomatologia de dores. Já em relação à realização de alongamentos após os exercícios, diferentes autores têm indicado que, embora sua realização promova uma sensação de bem-estar momentânea, sua prática parece não ser efetiva na prevenção do aparecimento de dores musculares tardias, nem tão pouco relacionadas às dores musculoesqueléticas crônicas (GENEEN et al., 2017).

Em relação à variável intervalo entre as séries, não foram encontradas diferenças entre as razões de prevalência de dor entre os sujeitos que adotavam um minuto ou menos tempo de intervalo com os que utilizavam tempo superior ao mencionado. Essa variável comumente tem sido empregada no TF na modulação da intensidade geral da sessão, influenciando diretamente em outras variáveis do treinamento como, por exemplo, a quantidade de carga e número de repetições executadas (FLECK e KRAEMER, 2017), e não repercutindo do relato de dor. Da mesma forma, a velocidade de execução de cada repetição não impactou na prevalência de dor nas três regiões analisadas. Estudos realizados com treinamentos de potência, principalmente em idosos, não têm relatado um comportamento diferenciado da dor, ou mesmo de lesões musculares neste tipo de treinamento quando comparados com velocidades mais tradicionais e lentas (TIGGEMANN et al., 2016).

Quanto à variável execução do exercício, puxada polia alta, e desenvolvimento, tanto na execução realizada pela frente quanto por trás da cabeça, não indicaram prevalências diferenciadas nos quadros de dores dos ombros e da região lombar. Kolber et al. (2010) ressaltam que o ombro é suscetível a dores e lesões no TF pela grande solicitação imposta durante os treinamentos, bem como pela posição desfavorável de execução, e, no caso especí- 
fico, com ombros rodados lateralmente (no caso das execuções realizadas por trás da cabeça). O presente estudo não analisou a realização desses exercícios com outras variáveis como, por exemplo, a quantidade de séries e cargas utilizadas, variáveis que poderiam potencializar os quadros de dores quando aqueles exercícios são realizados por trás da cabeça, ou seja, com os ombros rodados lateralmente. Mesmo assim, é importante salientar que o relato de dor é mais frequente em indivíduos que utilizam o membro superior em atividades repetitivas que impõem sobrecarga ao ombro, sobretudo acima de 90 graus de abdução ou flexão (MELLO et al., 2014), parecendo ser importante evitar volumes e intensidades excessivas durante a execução destes exercícios.

Considerando a amplitude articular durante a execução geral dos exercícios, em especial o que se relaciona à articulação dos joelhos nos exercícios agachamento/leg press, a razão de prevalência de dor entre os sujeitos que optavam por uma amplitude máxima comparado aos que a realizavam de forma reduzida foi semelhante. Em específico, sobre a amplitude articular dos joelhos durante os exercícios de agachamento/leg press, vários autores têm discutido nas últimas décadas a respeito da importância e os riscos associados à sua forma de execução (ESCAMILLA, 2001; LIST et al., 2013; HIRATA; DUARTE, 2007). Escamilla (2001) concluiu em seu estudo que, para atletas com joelhos saudáveis, a realização de amplitudes reduzidas seria recomendada pelo potencial risco de lesão dos meniscos e ligamentos cruzados e colaterais que aumentariam com uma maior profundidade do exercício. Já List et al. (2013) corroboram que o agachamento profundo promoveria uma maior amplitude dos joelhos, contudo, também promoveria menores alterações na coluna vertebral, reduzindo o stress na região lombar, além de promover uma menor flexão dos quadris (HIRATA; DUARTE, 2007). Assim, independente da forma de execução, o presente estudo não encontrou diferenças nas prevalências de dores entre as duas amplitudes de execução, e em específico ao exercício agachamento/leg press, considerando que ambos 
podem ser realizados desde que a individualidade do sujeito seja respeitada (MOURA et al., 2017).

Sujeitos que utilizaram cargas mais leves apresentaram maior prevalência de dor nos ombros quando comparados aos sujeitos que utilizaram cargas moderadas/pesadas durante seus treinamentos. A utilização de cargas menores é uma estratégia frequentemente utilizada para pessoas iniciantes e com baixos níveis de força, as quais possivelmente necessitam realizar mais força para a execução de determinadas tarefas do dia a dia, ficando assim mais expostas a lesões e dores musculoesqueléticas (FLECK; KRAEMER, 2017). Quadros crônicos de dores, associados à hipotrofia muscular, fraqueza ou lesão dos tecidos moles da região, em que a musculatura apresenta-se reduzida em sua seção transversa e demonstra hipotrofia seletiva de fibras e maior quantidade de gordura, podem ocasionar fraqueza e fadiga excessiva (COSTA; PALMA, 2005). Neste sentido, a utilização do TF tem sido uma estratégia no tratamento da redução de dor, tanto em sujeitos saudáveis (ZAVANELLA et al., 2012) como em pacientes com fibromialgia e artrite (AMBROSE; GOLIGHTLY, 2015). Em relação ao efeito da intensidade no comportamento da dor, não foram localizados artigos em que a comparação de diferentes cargas tenha sido realizada. Comparados ao grupo controle, Zavanella et al. (2012) identificaram uma redução significativa da dor de motoristas de ônibus após uma intervenção com TF com cargas moderadas/pesadas (10 - 12 repetições máximas). Mesmo assim, é importante salientar que a relação entre cargas leves e níveis baixos de força pode ser equivocada, sendo que alguns estudos têm falhado em associar diferentes níveis de força com dores ou desvios posturais, ou como tratamento analgésico (TIGGEMANN et al., 2018; AMBROSE; GOLIGHTLY, 2015).

As investigações científicas acerca das relações de causa e efeito, em especial nas situações de exposição em longo prazo (vários anos), sempre produzem um viés de confusão na sua interpretação. Assumir a relação direta de exposição de determinada variável do TF com as prevalências de dores pode ser um equívoco, e, 
portanto, deve ser interpretada com cautela. Além disso, é fundamental que se entenda que a dor crônica sempre possuirá causas multifatoriais, sendo tarefa difícil a de isolar sua causa principal. Outro aspecto limitante refere-se à perfeita compreensão dos questionamentos em relação às variáveis do TF pelos participantes, podendo gerar algum confundimento em seu preenchimento. Embora essas limitações devam ser consideradas no presente estudo, possivelmente a presente estratégia metodológica possa, pelo menos, indicar alguns pontos importantes para futuros estudos como, por exemplo, de um acompanhamento por meio de estudos de coorte, bem como agregar informações adicionais acerca do comportamento álgico e de lesões. Desta forma, conclui-se com o presente estudo que a prevalência de sujeitos com dores musculoesqueléticas é grande, sendo a região dos ombros, região inferior das costas e joelhos as mais acometidas. Além disso, a prevalência de dores entre os sujeitos que praticam o TF há mais de três anos e que utilizam cargas leves é maior quando comparadas com seus pares, sendo que outros aspectos como, por exemplo, amplitudes utilizadas, quantidade de séries e repetições, velocidades de execução e outras, não diferiram em suas prevalências.

\section{Referências}

AMBROSE, K.; GOLIGHTLY, Y. M. Physical exercise as non-pharma-
cological treatment of chronic pain: Why and when. Best Practice \& Research Clinical Rheumatology, EUA, v. 29, p. 120-130, 2015. AMERICAN COLLEGE OF SPORTS MEDICINE. Diretrizes do ACSM para os testes de esforço e sua prescrição. $10^{a}$ edição, Rio de Janeiro, Guanabara Koogan, 2018. 
BARONI, B. M. et al. Prevalência de alterações posturais em praticantes de musculação. Fisioterapia em Movimento, Curitiba, v. 23, n. 1, p. 129-139, 2010.

BARROS, E. N. C. Cross-cultural adaptation of the Nordic musculoskeletal questionnaire. International Nursing Review, EUA, v. 50, n. 2, p. 101-108, 2003.

BUTRAGUEÑO, J.; BENITO, P. J.; MAFULLI, N. Injuries in strength training: review and practical application. European Journal of Human Movement, Madrid, v. 32, p. 29-47, 2014.

COSTA, D.; PALMA, A. O efeito do treinamento contra resistência na síndrome da dor lombar. Revista Portuguesa de Ciências do Desporto, v. 2, n. 2, p. 224-234, 2005.

ESCAMILLA, R. F. Knee biomechanics of the dynamic. Medicine \& Science in Sports \& Exercise, EUA, v. 33, p. 127-141, 2001.

FLECK, S. J.; KRAEMER, W. J. Fundamentos do Treinamento de Força Muscular. $4^{a}$ edição, Editora Artmed, Porto Alegre, 2017.

GENEEN, L. J. et al. Physical activity ande exercise for chronic pain in adults: and overview of Cochrane Reviews (Review). Cochrane Database of Systematic Reviews, EUA, n. 4, 2017.

HIRATA, R. P.; DUARTE, M. Efeito da posição relativa do joelho sobre a carga mecânica interna durante o agachamento. Revista Brasileira de Fisioterapia, São Paulo, v. 11, n. 2, p. 121-125, 2007.

IBGE. Instituto Brasileiro de Geografia e Estática. Práticas de esporte na atividade física. 2017.

LEWIS, J. A Systematic Literature Review of the Relationship Between Stretching and Athletic Injury Prevention. Orthopaedic Nursing, EUA, v. 33, n. 6, p. 312-320, 2014.

LIST, R. et al. Kinematics of the trunk and the lower extremities during restricted and unrestricted squats. Journal of Strength and Conditioning Research, EUA, v. 27, n. 6, p. 1529-1538, 2013. 
KERR, Z. I. et al. Epidemiology of Weight Training-Related Injuries Presenting to United States Emergency Departments, 1990 to 2007. The American Journal of Sports Medicine, EUA, v. 38, n. 4, p. 765-771, 2010.

KOLBER, M. J. et al. Shoulder injuries attributed to resistance training: a brief review. Journal of Strength and Conditioning Research, EUA, v. 24, n. 6, p. 1696-1704, 2010.

MELLO, A. M. S. et al. Associação entre discinese escapular e dor no ombro em praticantes de musculação. Revista Brasileira de Ciências da Saúde, EUA, v. 18, n. 4, p. 309-314, 2014.

MOURA, A. G. et al. Aspectos biomecânicos do exercício agachamento profundo relacionados à articulação do joelho. Revista Científica Fagoc Saúde, Minas Gerais, v. 2, p.18-24, 2017.

OLIVA, O. J.; BANKOFF, A. D. P.; ZAMAI, C. A. Possíveis lesões musculares e ou articulares causadas por sobrecarga na prática da musculação. Revista brasileira Atividade Física e Saúde, Florianópolis, v. 3, n. 3, 1998.

OLIVEIRA, G. D.; CASA JúNIOR, A. J. Prevalência de lombalgia e avaliação da capacidade funcional lombar em praticantes de musculação. Estudos Vida e Saúde, Goiânia, v. 41, n. 2, p. 247258, 2014.

PEDERSEN, B. K.; SALTIN, B. Exercise as medicine - evidence for prescribing exercise as therapy in 26 different chronic diseases. Scandinavian Journal of Medicine \& Science in Sports, EUA, v. 25, n. 3, p. 1-72, 2015.

ROLLA, A. F. L. et al. O. Análise da percepção de lesões em academias de ginástica de Belo Horizonte: um estudo exploratório. Revista Brasileira de Ciência e Movimento, Brasília, v. 12, n. 2, p. 7-12, 2004.

SOUZA, G. L.; MOREIRA, N. B.; CAMPOS, W. Ocorrência e características de lesões entre praticantes de musculação. Revista Saúde e Pesquisa, Maringá, v. 8, n. 3, p. 469-477, 2015. 
TIGGEMANN, C. L. et al. Effect of traditional resistance and power training using rated perceived exertion for enhancement of muscle strength, power, and functional performance. AGE, EUA, v. 38, n. 42, p. 2-12, 2016.

TIGGEMANN, C. L. et al. The relationship between shoulder prothusion an neuromuscular variables in subject who practice strength training. International journal of environmental \& science education, EUA, v. 13, n. 7, p. 623-630, 2018.

ZAVANELLA, P. M. et al. Health and fitness benefits of a resistance training intervention performed in the workplace. Journal of Strength and Conditioning Research, EUA, v. 26, n. 3, p. 811817, 2012.

\title{
Notas
}

\section{Aprovação de comitê de ética em pesquisa}

pesquisa aprovada pelo Comitê de Ética do Centro Universitário UNIVATES. Título: Prevalência de dor musculoesquelética em praticantes de treinamento de força.

\section{Publisher}

\begin{abstract}
Universidade Federal de Goiás. Faculdade de Educação Física e Dança. Publicação no Portal de Periódicos UFG. As ideias expressadas neste artigo são de responsabilidade de seus autores, não representando, necessariamente, a opinião dos editores ou da universidade.
\end{abstract}

